\title{
Heterogeneous Doping to Improve the Performance of Thin Film Hematite Photoanodes for Solar Water Splitting
}

\author{
Asaf Kay, Daniel A. Grave, David S. Ellis, Hen Dotan, Avner Rothschild*
}

Department of Material Science and Engineering, Technion - Israel Institute of Technology, Haifa, Israel

*Email: avner@mt.technion.ac.il

\section{Published in ACS Energy Letters 2016 (DOI: 10.1021/acsenergylett.6b00287)}

\begin{abstract}
Ti-doped, undoped, and $\mathrm{Zn}$-doped hematite $\left(\alpha-\mathrm{Fe}_{2} \mathrm{O}_{3}\right)$ thick $(\sim 1 \mu \mathrm{m})$ films were found to be $n$-type, weak $n$-type, and $\boldsymbol{p}$-type, respectively. Heterogeneous doping profiles were generated in $30 \mathrm{~nm}$ thick hematite stacks on $\mathrm{F}: \mathrm{SnO}_{2}$ coated glass substrates with $25 \mathrm{~nm}$ thick $\mathrm{SnO}_{2}$ underlayers in order to investigate the effect of different doping profiles on photoelectrochemical performance and compare with homogenously-doped counterpart photoelectrodes. Among the homogenously-doped photoelectrodes, the Ti-doped sample displayed the highest plateau photocurrent but also the highest onset potential, whereas the Zn-doped one had the lowest onset potential and the lowest plateau photocurrent. Heterogeneouslydoped photoelectrodes displayed both high plateau photocurrent and low onset potential, with the highest performance achieved for the specimen with Ti-doped, undoped and Zn-doped layers at the bottom, center and top parts of the stack, respectively. This demonstrates the potential of heterogeneous doping to improve the performance of hematite photoelectrodes for solar water splitting.
\end{abstract}

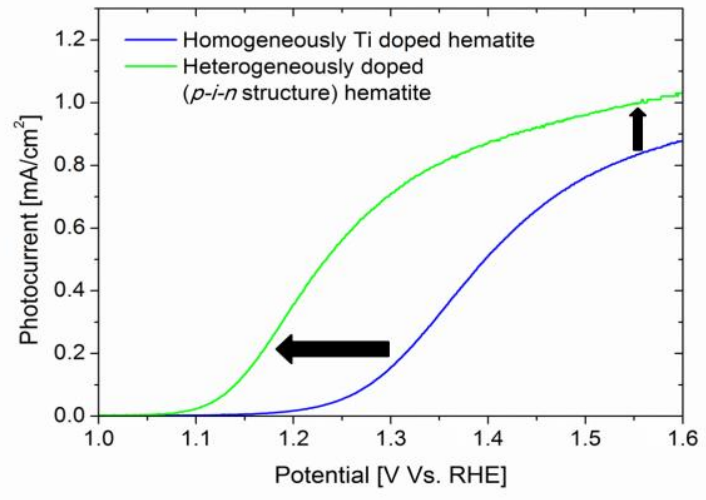

The most critical bottleneck towards the technological advancement of photoelectrochemical (PEC) cells for use in solar water splitting is the photoelectrode material, which must meet the requirements of being inexpensive, efficient, robust, and stable for water photoelectrolysis. One of the most promising materials for use as a photoanode for water splitting is hematite $\left(\alpha-\mathrm{Fe}_{2} \mathrm{O}_{3}\right)$ due to its vast abundance, low cost, light absorption characteristics and stability in the conditions needed for water oxidation. ${ }^{1}$ Indeed, a reported working stability of at least 1000 hours has very recently been reported. ${ }^{2}$ But hematite photoanodes also display some prominent 
disadvantages that are thought to arise from low electron mobility $\left(\sim 0.1 \mathrm{~cm}^{2} \mathrm{~V}^{-1} \mathrm{~s}^{-1}\right)^{3,4}$ and short lifetime $(\sim 100$ ps $)^{5}$ and diffusion length $(\sim 2-5 \mathrm{~nm})^{4,6}$ of photo-generated minority charge carriers, i.e., holes. This results in massive bulk recombination that reduces the photocurrent. Undoped hematite is highly resistive ${ }^{7}$ and therefore hematite photoanodes are usually doped with donors such as $\mathrm{Sn}, \mathrm{Si}$ or Ti in order to increase their conductivity. ${ }^{8}$ Another reason for doping hematite photoanodes is in order to enhance the photovoltage. However, a high doping level often reduces the lifetime of minority charge carriers due to enhanced recombination with majority charge carriers. ${ }^{9}$ This gives rise to a dilemma as to the optimal doping strategy for high performance hematite photoanodes.

This work explores heterogeneous doping as a means to improve the performance of hematite photoelectrodes. Our approach is inspired by PV devices such as amorphous Si solar cells, ${ }^{10}$ wherein $p$-i-n stacks are often employed in order to achieve optimal light harvesting and charge generation with minimal recombination in the low-doped ( $i$ ) layer, and charge separation through selective collection of electrons in the donor-doped $(n)$ layer and holes in the acceptor-doped $(p)$ layer. ${ }^{11}$ Another approach is to employ graded doping profiles which has shown promising results in $\mathrm{W}$-doped $\mathrm{BiVO}_{4}$ photoanodes. ${ }^{12}$ For hematite-based water splitting, $n-\mathrm{Fe}_{2} \mathrm{O}_{3} / p-\mathrm{NiO}$ and $n-\mathrm{Fe}_{2} \mathrm{O}_{3} / p-\mathrm{CaFe}_{2} \mathrm{O}_{4}$ heterojunction photoelectrodes have exhibited improved efficiencies. ${ }^{13,14}$ Lin et al. investigated homojunction hematite photoelectrodes, where $p$-type $\mathrm{Mg}$-doped $\mathrm{Fe}_{2} \mathrm{O}_{3}$ was deposited on top of $n$-type $\mathrm{Fe}_{2} \mathrm{O}_{3}$ by atomic layer deposition (ALD), resulting in a reduction of the the onset potential by approximately $200 \mathrm{mV} .{ }^{15}$ Here, we study heterogeneous doping profiles in thin film hematite photoelectrodes on F: $\mathrm{SnO}_{2}$ (FTO) coated glass substrates with an $\mathrm{SnO}_{2}$ underlayer. All the films were deposited by pulsed laser deposition (PLD), which is known for precise stoichiometry transfer from target to film as well as excellent structural and thickness control and high reproducibility. ${ }^{16}$ Thus, PLD is a powerful tool for this study, as demonstrated in another study in which we compared different dopants in thin film hematite photoanodes. ${ }^{8}$ First, homogenous Ti-doped, undoped, and Zn-doped thick $(\sim 1 \mu \mathrm{m})$ films were identified with conclusive evidence to be $n$-type, weak $n$-type, and $p$-type semiconductors, respectively, and are thus termed $n, i$ (intrinsic), and $p$ throughout this work. Subsequently, thin $(30 \mathrm{~nm})$ film photoelectrodes with homogeneous or heterogeneous doping profiles were investigated, are shown in Figure 1. We show that using a combination of these layers in order to create heterogeneous doping profiles, especially a $p-i-n$ stack, can significantly improve the photoelectrochemical performance. 
a

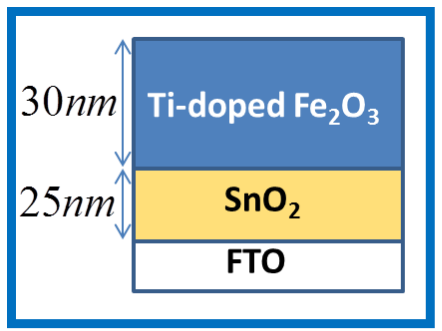

d

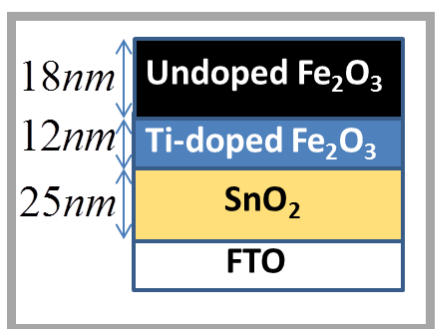

b

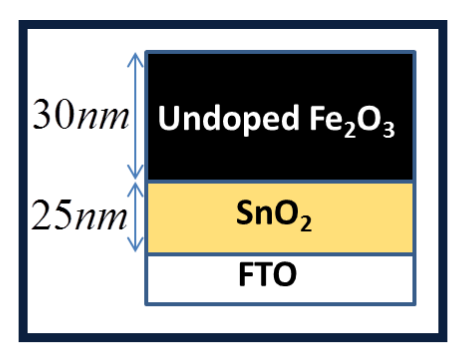

e

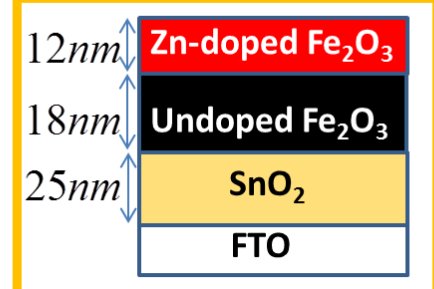

C

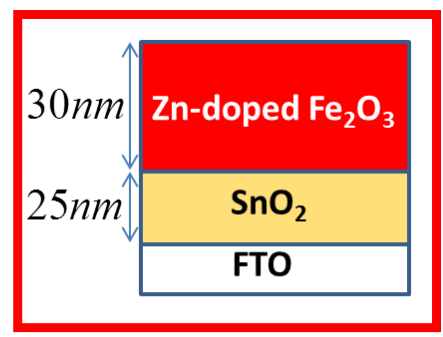

f

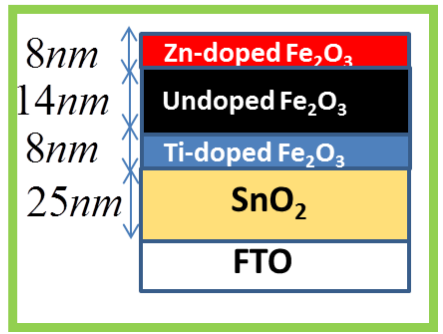

Figure 1. Thin film hematite photoelectrode configurations investigated in this work: (a) Homogeneously Ti-doped ( 1 cation\%) hematite film; (b) Undoped hematite film; (c) Homogeneously Zn-doped ( 1 cation\%) hematite film; (d) Heterogeneously doped $i-n$ stack; (e) Heterogeneously doped $p-i$ stack; and (f) Heterogeneously doped $p-i-n$ stack.

All 6 photoelectrodes that were studied here were comprised of a $30 \mathrm{~nm}$ thick stack of hematite films with either homogeneous or heterogeneous doping profiles, as illustrated in Figure 1. The hematite stacks were deposited on FTO-coated glass substrates with $25 \mathrm{~nm}$ thick undoped $\mathrm{SnO}_{2}$ underlayers. The hematite and $\mathrm{SnO}_{2}$ films were deposited by PLD. Figure S1 presents a photograph of the photoelectrodes. The microstructure and crystallinity of the films was examined by HRSEM and XRD, and all of the photoelectrodes displayed polycrystalline morphology, with hematite Bragg reflections superimposed on the $\mathrm{SnO}_{2}$ reflections from the substrate (see Figures S2 and S3). Transmittance $(T)$ and reflectance $(R)$ spectra of the photoelectrodes were measured using a spectrophotometer equipped with an integrating sphere, and the absorptance $(A)$ was calculated using $A=1-T-R$. Figure 2 presents the absorptance spectra of all of the photoelectrodes. There are some differences in the absorptance spectra of different photoelectrodes but they are too small to account for the differences in the PEC performance of the respective photoelectrodes. 


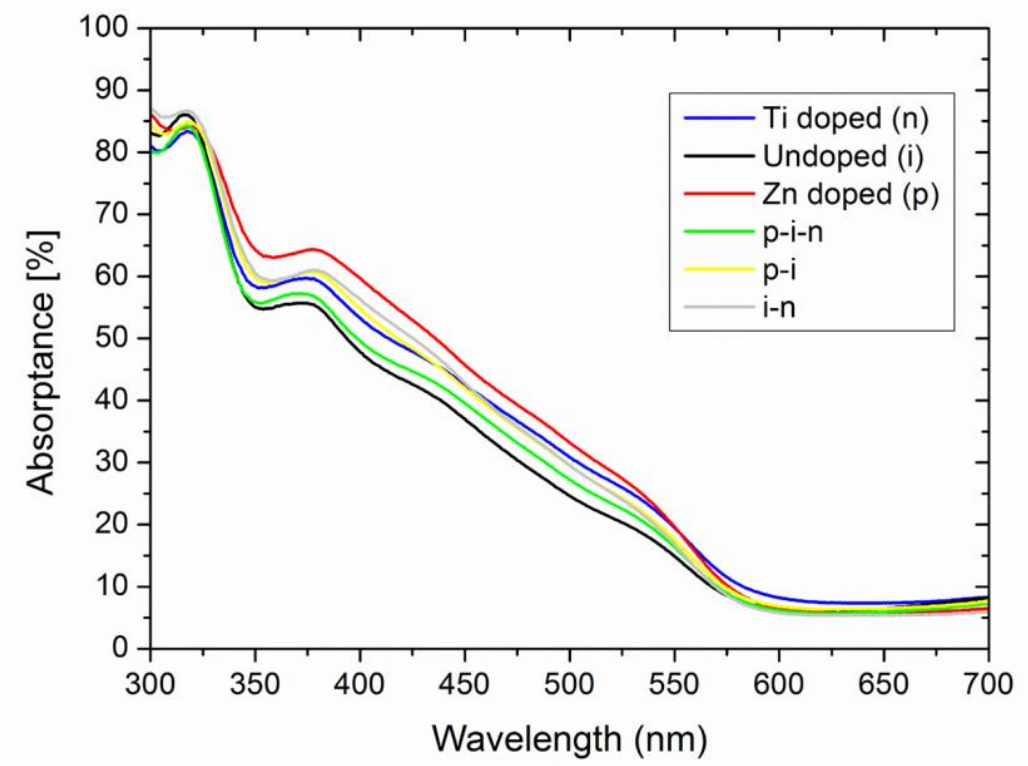

Figure 2. Absorptance spectra of all of the thin film photoelectrodes.

The absorbed photocurrent density, $J_{a b s,}$ was calculated using:

$$
J_{a b s}=q \int_{300 n m}^{590 n m} \Phi_{(\lambda)} A_{(\lambda)} d \lambda
$$

where $q$ is the electron charge, $\Phi_{(\lambda)}$ is the irradiance spectrum (expressed in units of photon flux, photons $/ \mathrm{cm}^{2} / \mathrm{s} / \mathrm{nm}$ ) of the light source that was used for the PEC measurements (see Figure S4), and $A_{(\lambda)}$ is the absorptance spectrum of the respective specimen (from Figure 2). Equation (1) assumes that all the photons in this wavelength range were absorbed in the hematite stack, ignoring wasted absorption elsewhere in the specimen. Thus, it provides an upper limit estimation of $J_{a b s}$. Table 1 lists the calculated values of $J_{a b s}$ for the photoelectrodes from Figure 2. They are within $\pm 12 \%$ of the average $J_{a b s}$ for all of the specimens.

Table 1. Calculated $J_{a b s}$ for the thin film photoelectrodes in Figure 1.

\begin{tabular}{|c|c|c|c|c|c|c|}
\hline Sample & $\begin{array}{c}\text { Ti-doped } \\
(\boldsymbol{n})\end{array}$ & $\begin{array}{c}\text { Undoped } \\
(\boldsymbol{i})\end{array}$ & $\begin{array}{c}\text { Zn-doped } \\
(\boldsymbol{p})\end{array}$ & $\boldsymbol{p}$-i-n & $\boldsymbol{p}-\boldsymbol{i}$ & $\boldsymbol{i}-\boldsymbol{n}$ \\
\hline $\begin{array}{c}\boldsymbol{J}_{\text {abs }} \\
\left(\mathbf{m A} / \mathbf{c m}^{2}\right)\end{array}$ & 4.1 & 3.4 & 4.3 & 3.7 & 3.9 & 3.9 \\
\hline
\end{tabular}

As a preliminary step towards the investigation of heterogeneous doping profiles in thin film photoelectrodes, the effect of different dopants in homogenously-doped thick films was examined. According to the defect chemistry of $\mathrm{Fe}(\mathrm{III})_{2} \mathrm{O}_{3}, \mathrm{Zn}$ (II) is expected to be an acceptor and Ti(IV) a donor. ${ }^{17}$ Undoped hematite is expected to be slightly $n$-type due to oxygen vacancies. In order to confirm these expectations, surface photovoltage (SPV) measurements 
were performed to determine the conductivity type ( $n$ - or $p$-type) of Ti-doped, Zn-doped and undoped thick $(\sim 1 \mu \mathrm{m})$ hematite films deposited on FTO without an $\mathrm{SnO}_{2}$ underlayer. Figure 3(a) shows the contact potential difference (CPD), i.e., the difference between the work functions of the sample and the probe tip, in the dark and under illumination. The work function of semiconductors depends on their electron affinity, the position of the Fermi energy within the band gap, and the band bending at the surface. The surface band bending is typically positive (upward) for $n$-type semiconductors and negative (downward) of $p$-type semiconductors, thereby increasing or decreasing the work function, respectively. ${ }^{18}$ Without knowledge of the band bending values, CPD measurements in the dark cannot indicate the relative doping levels of different specimens. The response of the CPD to illumination, i.e., the surface photovoltage (SPV), can be understood by referring to Figure 3(b), which shows hypothetical band diagrams of weak $n$-type, $n$-type and $p$-type semiconductors. Upon illumination, photo-generated charge carriers will redistribute towards or away from the surface, in such a way as to reduce the magnitude of the surface charge, and hence the band bending. Therefore, since the undoped and Ti-doped hematite specimens display a negative change in the CPD under illumination, they are $n$-type semiconductors, whilst the $\mathrm{Zn}$-doped sample displays the opposite effect, indicating $p$-type behavior. ${ }^{18}$ The magnitude of the SPV must be interpreted with caution. ${ }^{19}$ Higher doping levels exhibit smaller band bending for the same amount of surface charge, ${ }^{20,21}$ which would seem to be consistent with the much larger SPV observed for the undoped sample as compared to the Ti-doped sample. On the other hand, doped samples are predicted to have a higher adsorption of charged molecules on the surface, ${ }^{22}$ which would impact the band bending in the opposite way. Resistance measurements indicate low carrier concentration for the undoped sample, so we can conclude that it is weak $n$-type, and will henceforth refer to it as an intrinsic $(i)$ semiconductor, in analogy to $p-i-n$ thin film solar cells.

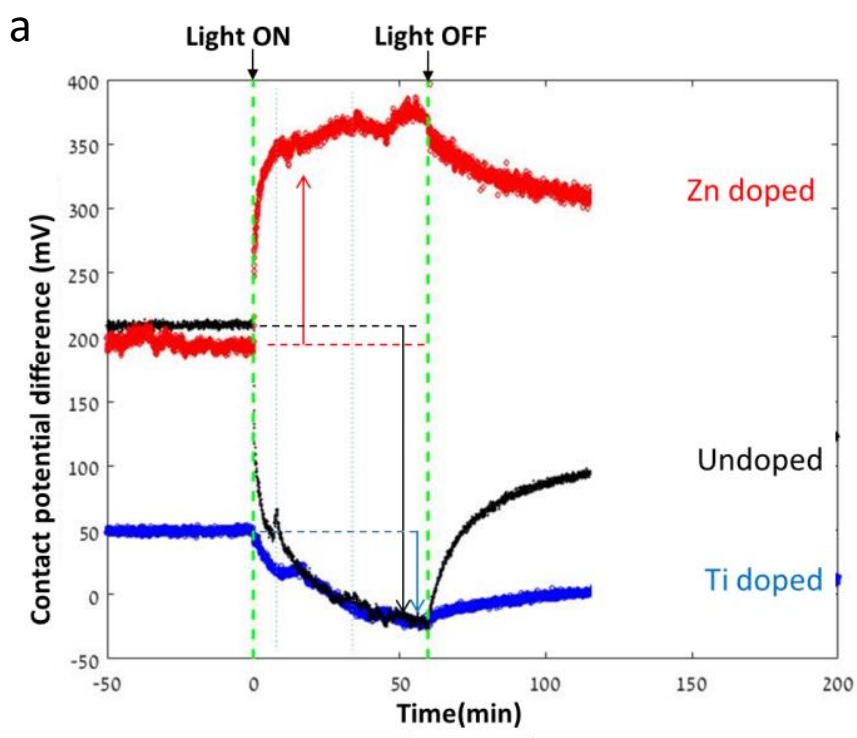

b

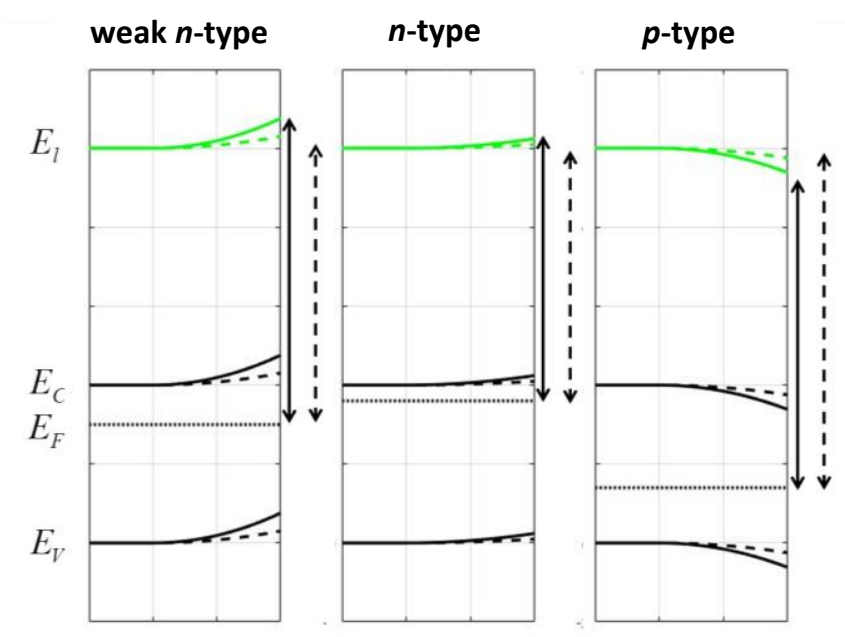

Figure 3. (a) Surface photovoltage measurements of homogeneously-doped and undoped hematite thick films upon illumination with white light between $\mathrm{t}=0$ and $60 \mathrm{~min}$. (b) Hypothetical band diagram for weak $n$-type (left), $n$-type (middle), and $p$-type (right) semiconductors in dark (solid lines) and under illumination (dashed lines). The solid and dashed vertical arrows represent the work function in the dark and under illumination, respectively. For simplicity the SPV was arbitrarily assumed to be $60 \%$ of the band bending in all cases, and possible surface dipoles are not accounted for. $E_{l}, E_{C}, E_{F}$, and $E_{V}$ denote the local vacuum level, conduction band edge, Fermi energy and valence band edge, 
respectively.

To complement the SPV measurements and measure the majority carrier concentration and mobility, Hall effect measurements were carried out for $\sim 140 \mathrm{~nm}$ thick hematite epilayers deposited on single crystal (0001) sapphire substrates in order to avoid spurious grain boundary effects. ${ }^{23}$ The films were deposited from the same targets from which the respective photoelectrodes were prepared, therefore it is safe to assume that the intrinsic bulk behavior of the hematite films would be similar in both cases. In agreement with the SPV results, the Hall effect measurements show that the $\mathrm{Zn}$-doped hematite film displays $p$-type behavior whereas the Ti-doped film exhibits an $n$-type behavior. The charge carrier (hole) concentration and Hall mobility in the $\mathrm{Zn}$-doped hematite film were $2.0 \times 10^{14}$ $\mathrm{cm}^{-3}$ and $6.1 \mathrm{~cm}^{2} \mathrm{~V}^{-1} \mathrm{~s}^{-1}$, respectively, whereas the Ti-doped hematite film had a charge carrier (electron) concentration of $4.6 \times 10^{18} \mathrm{~cm}^{-3}$ and Hall mobility of $0.1 \mathrm{~cm}^{2} \mathrm{~V}^{-1} \mathrm{~s}^{-1}$. The resistance of the undoped hematite film was too high for a Hall effect measurement. The charge carrier concentration and Hall mobility of our Ti-doped hematite film are similar to values reported previously for Ti-doped hematite films deposited by molecular beam epitaxy (MBE) ${ }^{24}$ As far as we know, no Hall effect data were reported for $p$-type hematite. The carrier concentration in our films is 4 orders of magnitude lower for the $p$-type $\mathrm{Zn}$-doped hematite film as compared to the $n$-type Ti-doped film, and the hole mobility is found to be almost two orders of magnitude larger than the electron mobility. The hole concentration in our $\mathrm{Zn}$-doped thick film is lower by one or two orders of magnitude compared to previous reports on Mg-doped hematite photoelectrodes. ${ }^{15,25}$ It is noteworthy that there is some dispute as to whether the mobility and carrier concentration values extracted from Hall effect measurements are accurate for small-polaron conductors. ${ }^{26}$ Nevertheless, the Hall effect measurements confirm the assignment of $n$ - and $p$-type behavior to the Ti- and Zn-doped hematite films, respectively. Further evidence for the assignment of weak $n$-type, $n$-type and $p$ type conductivities to the undoped, Ti-doped and Zn-doped thick $(\sim 1 \mu \mathrm{m})$ hematite films on FTO-coated glass substrates was obtained by Mott-Schottky analysis of capacitance vs. potential measurements, see Figure S5. The Ti-doped specimen displays linear Mott-Schottky plots at different frequencies, but the Zn-doped and undoped specimens display curved plots that cannot be analyzed quantitatively without an elaborate physical model. Nevertheless, the sign of the slopes corresponds to $n$-type, $p$-type and $n$-type semiconductors for the Ti-doped, $\mathrm{Zn}$ doped and undoped specimens, respectively.

The photoelectrochemical performance of all of the thin $(30 \mathrm{~nm})$ film photoelectrodes was examined by voltammetry measurements in the dark and under solar-simulated light. The results are shown in Figure 4. Figure 4a shows the photocurrent, $J_{\text {photo }}$, obtained by subtracting the dark current from the current measured under solarsimulated light (see Figure S6), as a function of the applied potential. The photocurrent in Figure 4a was measured in alkaline aqueous electrolyte solution $(1 \mathrm{M} \mathrm{NaOH}$ in deionized water) with no sacrificial reagents. Figure $4 \mathrm{~b}$ shows the photocurrent obtained with a hole scavenger $\left(\mathrm{H}_{2} \mathrm{O}_{2}, 0.5 \mathrm{M}\right)$ in the alkaline solution $(1 \mathrm{M} \mathrm{NaOH}$ in deionized water). Interestingly, the Zn-doped hematite photoelectrode displayed an ambipolar response with relatively high photoanodic currents at potentials above $0.8 \mathrm{~V}_{\mathrm{RHE}}$ and low photocathodic currents below $0.8 \mathrm{~V}_{\mathrm{RHE}}$, see Figure $\mathrm{S} 7$. A thick $(\sim 1 \mu \mathrm{m})$ film counterpart photoelectrode of the same structure and composition displayed only photocathodic currents, without photoanodic currents (see Figure S8), as expected for a $p$-type photoelectrode. Thus, the photoanodic response of the thin film $\mathrm{Zn}$-doped hematite photoelectrode suggests that the conductivity type was 
inverted from $p$-type to $n$-type, probably due to electron injection from the FTO current collector electrode. A similar $p$-type to $n$-type inversion was observed in Fe-doped $\mathrm{SrTiO}_{3}$ thin films on $\mathrm{Nb}$-doped $\mathrm{SrTiO}_{3}$ ( $n$-type) substrates, as reported elsewhere. ${ }^{27}$ Domen et al. reported a similar effect in $\mathrm{La}_{5} \mathrm{Ti}_{2} \mathrm{CuS}_{5} \mathrm{O}_{7}$ photoelectrodes that displayed ambipolar photocurrents depending on the current collector electrode on which they were deposited. ${ }^{28}$

a

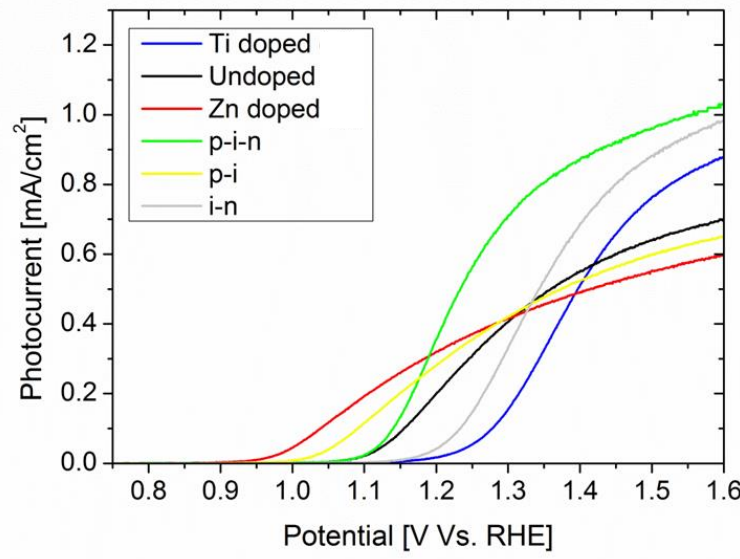

b

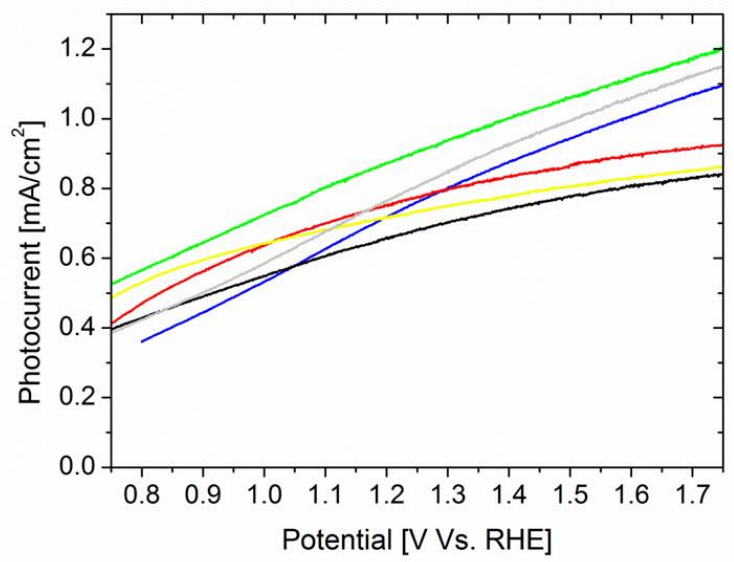

Figure 4. Photocurrent-potential $\left(J_{\text {photo }}-U\right)$ voltammograms measured in aqueous alkaline electrolyte solution (1M $\mathrm{NaOH}$ in deionized water); (a) without sacrificial reagents, and (b) with a hole scavenger $\left(0.5 \mathrm{M} \mathrm{H}_{2} \mathrm{O}_{2}\right)$.

Of all the photoelectrodes in this study, the Zn-doped one (red curve) displayed the lowest onset potential, but also the lowest plateau photocurrent at high potentials (>1.4 $\mathrm{V}_{\mathrm{RHE}}$ ). For the homogenously-doped photoelectrodes the trends are clear: going from $\mathrm{Zn}$-doped, to undoped to Ti-doped films increases the onset potential and increases the plateau photocurrent. Keeping in mind that it is desired to have both low onset potential and high photocurrent, the results in Figure 4(a) show that both of these merits are difficult to achieve simultaneously in homogenously-doped hematite photoelectrodes because one comes at the expense of the other. However, the dilemma between low potential and high photocurrent can be resolved by going from homogenous to heterogeneous doping. Thus, the $p-i-n$ stack (green curve) reaches high photocurrent $(>1$ $\mathrm{mA} / \mathrm{cm}^{2}$ at $1.6 \mathrm{~V}_{\mathrm{RHE}}$ ) and has a rather low onset potential of $1.05 \mathrm{~V}_{\mathrm{RHE}}$, which is in between the lowest onset potential that was achieved by the $\mathrm{Zn}$-doped photoelectrode $\left(0.95 \mathrm{~V}_{\mathrm{RHE}}\right)$ and the highest onset potential that was achieved by the Ti-doped photoelectrode $\left(1.15 \mathrm{~V}_{\mathrm{RHE}}\right)$. On top of having the best compromise between high photocurrent and low potential, the $p-i-n$ stack also displays a high fill factor which is shown by the steep rise of the photocurrent. The absorbed photon to current efficiency (APCE $=J_{\text {photo }} / J_{a b s}$ ) was calculated using the $J_{\text {photo }}$ values at $1.48 \mathrm{~V}_{\mathrm{RHE}}$ (Figure $4 \mathrm{a}$ ) and $J_{a b s}$ values from Table 1, and the results are presented in Table 2. The $p$-i-n stack reached an APCE of $26 \%$ at $1.48 \mathrm{~V}_{\mathrm{RHE}}$, placing it among the most efficient thin film hematite photoelectrodes reported to date ${ }^{29}$ with respect to the internal quantum efficiency (IQE), i.e., the ability to convert absorbed photons to photocurrent. This shows, indirectly, the superior charge separation and collection efficiency of the heterogeneously-doped stacks, especially the $p-i-n$ one. 
Table 2. APCE at $1.48 \mathrm{~V}_{\text {RHE. }}$

\begin{tabular}{|c|c|c|c|c|c|c|}
\hline Sample & Ti-doped & Undoped & Zn-doped & $\boldsymbol{p}$-i-n & $\boldsymbol{p}-\boldsymbol{i}$ & $\boldsymbol{i}-\boldsymbol{n}$ \\
\hline APCE & $18.3 \%$ & $18.5 \%$ & $12.5 \%$ & $26 \%$ & $14.8 \%$ & $21.6 \%$ \\
\hline
\end{tabular}

To confirm this indirect observation more directly, we carried out complementary voltammetry measurements in the presence of a hole scavenger $\left(\mathrm{H}_{2} \mathrm{O}_{2}\right)$ in the electrolyte solution. As reported elsewhere, these measurements enable probing the photoelectrochemical properties of hematite photoelectrodes without the added complexity of the four-electron water oxidation reaction. ${ }^{30}$ The results are presented in Figure 4(b), showing the photocurrent vs. potential curves in the presence of the hole scavenger $\left(0.5 \mathrm{M} \mathrm{H}_{2} \mathrm{O}_{2}\right.$ and $1 \mathrm{M} \mathrm{NaOH}$ in deionized water). One can see two types of behavior: one for all the stacks containing a Ti-doped ( $n$-type) hematite layer (green, grey and blue curves), and another one for all the other stacks without it (red, yellow and black curves). The former group of photoelectrodes displays a steep rise in the $\mathrm{H}_{2} \mathrm{O}_{2}$ photocurrent as a function of the applied bias, and the latter one shows slow rise that saturates at high potentials. This is an interesting observation that requires further investigation in order to understand the underlying physics. As expected, the $p$ - $i-n$ stack displays the highest $\mathrm{H}_{2} \mathrm{O}_{2}$ photocurrent from all the photoelectrodes. This is also the case after normalizing the $\mathrm{H}_{2} \mathrm{O}_{2}$ photocurrent by $J_{a b s}$ (from Table 1, see Figure S9). This result confirms the superior charge separation and collection efficiency of the $p-i-n$ stack with respect to all the other photoelectrodes that were studied here.

To compare the PEC performance benchmarks from Figure 4(a), the photoelectrodes are ranked in the following order from best to worst in terms of the onset potential: Zn-doped film $>p-i$ stack $>p-i-n$ stack > undoped film $>i$ - $n$ stack $>$ Ti-doped film. The results suggest that having a Zn-doped hematite layer at the front interface with the aqueous electrolyte solution significantly reduces the onset potential. This suggests that this layer suppresses the surface recombination between electrons and holes. However, in terms of the plateau photocurrent achieved at $1.48 \mathrm{~V}_{\mathrm{RHE}}$, the photoelectrodes are ranked as follows: $p-i-n$ stack $>i-n$ stack $>$ Tidoped film $>$ undoped film $>p-i$ stack $>$ Zn-doped film, suggesting that having a Ti-doped hematite layer at the back interface increases the photocurrent at high potentials where the barrier for charge transfer from the photoelectrode to the electrolyte is small. This implies a good contact between Ti-doped ( $n$-type) hematite and the $\mathrm{SnO}_{2} / \mathrm{FTO}$ current collector. Indeed, Ti-doped hematite was previously found to exhibit ohmic behavior with FTO whereas the contact with undoped hematite displayed non-ohmic behavior. ${ }^{31}$ Another beneficial effect of having an $n$-type layer at the backside of the stack is that it serves as a selective filter for electrons, thereby enhancing the charge carrier separation within the stack. ${ }^{11}$ Likewise, a $p$-type layer at the front side of the stack serves as a selective filter for holes. Thus, the $p-i-n$ stack achieved the highest plateau photocurrent of all the photoelectrodes examined in this study by inducing an assymetry for charge transport through the stack.

In summary, Ti-doped, undoped and $\mathrm{Zn}$-doped hematite thick films were found to display characteristics 
of $n$-type, weak $n$-type and $p$-type semiconductors, respectivly. The Ti-doped thin film hematite photoelectrode dispalyed the highest plateau photocurrent at high potentials but also the highest onset potential, whereas the Zn-doped counterpart photoelectrode dispalyed the opposite behavior with the lowest onset potential but also the lowest plateau photocurrent of all the photoanodes examined here. This shows that for homogenouslydoped hematite photoelectrodes there is a trade-off between photocurrent and potential. This trade-off can be resolved by going from homogenous to heterogenous doping profiles, as demonstrated by the $\mathrm{Zn}$-doped / undoped / Ti-doped ( $p-i-n)$ stack, that achived the highest plateau photocurrent and one of the lowest onset potential of all the photoelectrodes examined in this study. This work shows that heterogeneous doping profiles can significantly improve the performance of thin film hematite photoelectrodes, similarly to other thin film photovoltaic devices such as amorphous Si PV cells with $p$-i-n structure.

\section{ASSOCIATED CONTENT}

\section{Experimental Methods}

Photoelectrode fabrication. Thin film hematite photoelectrodes were prepared by depositing $30 \mathrm{~nm}$ thick hematite stacks and $25 \mathrm{~nm}$ thick $\mathrm{SnO}_{2}$ underlays on fluorinated tin oxide (FTO) coated soda-lime glass substrates (TEC 15, Pilkington, Tokyo, Japan). The hematite and $\mathrm{SnO}_{2}$ films were deposited by pulsed laser deposition (PLD) system using a turn-key 2" PLD Workstation (Surface Systems \& Technology, GmbH) equipped with a $\mathrm{KrF}(\lambda=248 \mathrm{~nm})$ excimer laser (COMPexPro 102, Coherent). The $\mathrm{SnO}_{2}$ underlayer was deposited prior to the hematite deposition using 400 laser pulses $(\sim 25 \mathrm{~nm})$ and a substrate to target distance of $75 \mathrm{~mm}$. Subsequently, the hematite films were deposited using 5000 laser pulses and a substrate to target distance of $70 \mathrm{~mm}$. The laser repetition rate was $3 \mathrm{~Hz}$ and the heater set point was $500^{\circ} \mathrm{C}$ which corresponds to a substrate temperature of approximately $450^{\circ} \mathrm{C}$. At this temperature Sn diffusion from the substrate into the hematite film is negligible, as reported elsewhere. ${ }^{32}$ All depositions were carried out in an $\mathrm{O}_{2}$ gas atmosphere at a constant pressure of $25 \mathrm{mTorr}$. The TEC15 substrates were cut to $15 \mathrm{~mm}$ x $30 \mathrm{~mm}$ pieces and cleaned with isopropanol and deionized water prior to the deposition process. The films were deposited from targets produced by solid state reaction route using high purity (99.99\% Alfa Aesar) powders of the base (binary) oxides: $\mathrm{Fe}_{2} \mathrm{O}_{3}, \mathrm{TiO}_{2}$, and $\mathrm{ZnO}$. The based powders were weighted and mixed to achieve the desired stoichiometry (undoped $\mathrm{Fe}_{2} \mathrm{O}_{3}$ and $\mathrm{Fe}_{2} \mathrm{O}_{3}$ doped with 1 cation\% of Ti or $\mathrm{Zn}$ ). The mixture was ball-milled, pressed, and sintered in air at $1200^{\circ} \mathrm{C}$, yielding 1" disk-shaped pellets. The chemical composition of the targets were examined by EDS, confirming the expected composition with nearly 1 cation\% Ti or $\mathrm{Zn}$, as reported elsewhere.

Microstructural characterizations. The crystallinity and phase composition of the photoelectrodes was examined by grazing incident X-ray diffraction (GIXRD), using a Rigaku SmartLab diffractometer. The acquisition conditions were parallel beam configuration with $\mathrm{Cu} K \alpha$ radiation in the $2 \theta$ range of $20-75^{\circ}$ at a scan rate of $0.01^{\circ} \mathrm{s}^{-1}$. The results, presented in Figure S2, show Bragg reflections of the $\alpha-\mathrm{Fe}_{2} \mathrm{O}_{3}$ hematite and $\mathrm{SnO}_{2}$ rutile phases, with no other phases detected. The crystalline domain size was evaluated by applying the 
Scherrer equation to the peak broadening of the hematite (110) Bragg reflections (Table S1). The surface morphology of the photoanodes was examined by high-resolution scanning electron microscopy (HRSEM) using a Zeiss Ultra Plus filed-emission HRSEM. Exemplary micrographs taken at a magnification of 100000, working distance of $3.3 \mathrm{~mm}$ and electron acceleration voltage of $4 \mathrm{kV}$ as presented in Figure $\mathrm{S} 3$, showing similar surface morphology for all the specimens.

Electrical, electrochemical and optical characterizations. In order to determine the conductivity type of the doped and undoped hematite films, surface photovoltage measurements were carried out (in ambient conditions) using a Kelvin probe (KP Technologies) with a $5 \mathrm{~mm}$ Ti tip and white light source. To that end, Ti-doped, Zn-doped and undoped hematite thick films were deposited directly on TEC15 substrates without $\mathrm{SnO}_{2}$ underlayers and using 200,000 pulses instead of 5000 pulses for the thin film photoelectrodes. This was done in order to reduce the effect of the back contact. To measure the majority carrier concentration and mobility, Hall effect measurements were carried out for heteroepitaxial hematite films (doped with Ti or Zn, 1 cation\%) that were deposited directly on $c$-plane sapphire substrates, as reported elsewhere. ${ }^{23}$ This was done to avoid spurious grain boundary effects. The undoped specimen could not be measured due to its high resistance. The films were fitted with indium point contacts at the corners, and 4-point-probe (4PP) conductivity measurements were carried out using KEITHLEY 2635 SourceMeter and KEITHLEY 6514 electrometer and a $1 \mathrm{~T}$ magnet. Capacitance vs. potential measurements were carried out on Ti-doped, Zn-doped and undoped thick $(\sim 1 \mu \mathrm{m})$ film photoelectrodes. The measurements were carried out in alkaline aqueous electrolyte solutions (1 $\mathrm{M} \mathrm{NaOH}$ in deionized water) without sacrificial reagents. The results are presented in Figure S5. Optical reflection and transmission spectra were measured using a spectrophotometer (Agilent Cary 5000) equipped with an integrating sphere.

Photoelectrochemical measurements. The photoelectrochemical performance of the thin film photoelectrodes was examined by three electrode voltammetry measurements with the photoanode serving as the working electrode and a platinum wire as the counter electrode. $\mathrm{An} \mathrm{Ag} / \mathrm{AgCl}$ electrode in saturated $\mathrm{KCl}$ solution served as the reference electrode. The photoelectrochemical measurements were carried out in alkaline aqueous electrolyte solutions (1 M NaOH in deionized water) without sacrificial reagents (Figure 4a). Complementary measurements (Figure 4b) were carried out with a hole scavenger $\left(0.5 \mathrm{M} \mathrm{H}_{2} \mathrm{O}_{2}\right)$ added to the $1 \mathrm{M} \mathrm{NaOH}$ solution. Current versus potential $(J-U)$ linear sweep voltammograms were acquired both in the dark and in front light illumination (from the film side) using a solar simulator (ABET Technologies Sun 3000 class AAA solar simulator). The irradiance spectrum of the solar simulator is presented in Figure S4.

\section{Supporting Information}

Photograph of the thin film hematite photoelectrodes; Grazing incident X-ray diffraction (GIXRD) patterns and HRSEM micrographs of the photoelectrodes; Irradiance spectrum of the solar simulator; Mott-Schottky plots of thick $(\sim 1 \mu \mathrm{m})$ film hematite photoelectrodes; Dark, light and chopped-light voltammograms of thin $(30 \mathrm{~nm})$ and thick $(\sim 1 \mu \mathrm{m})$ film Zn-doped hematite photoelectrodes; Dark and light voltammograms and (APCE) vs. potential curves of thin $(30 \mathrm{~nm})$ film hematite photoelectrodes with different doping profiles; Crystalline 
domain size of the thin film hematite photoelectrodes; and Hall effect data for $~ 140 \mathrm{~nm}$ thick epitaxial hematite films doped with Ti or Zn.

\section{AUTHOR INFORMATION}

\section{Corresponding Author}

* Email: avner@mt.technion.ac.il ; Phone: +972-(0)4-8294576

\section{ACKNOWLEDGMENTS}

This research has received funding from the European Research Council under the European Union's Seventh Framework programme (FP/200702013) / ERC Grant agreement n. [617516]. The results were obtained using central facilities at the Technion's Hydrogen Technologies Research Laboratory (HTRL), supported by the Adelis Foundation, the Nancy \& Stephen Grand Technion Energy Program (GTEP) and by the Solar Fuels ICORE program of the Planning and Budgeting Committee and the Israel Science Foundation (Grant n. 152/11); the Photovoltaic Laboratory, supported by the Nancy \& Stephen Grand Technion Energy Program (GTEP) and by the Russell Berrie Nanotechnology Institute (RBNI); and the Micro and Nano Fabrication Unit (MNFU).

\section{References}

(1) Sivula, K.; Le Formal, F.; Grätzel, M. Solar Water Splitting: Progress Using Hematite $\left(\alpha-\mathrm{Fe}_{2} \mathrm{O}_{3}\right)$ Photoelectrodes. ChemSusChem 2011, 4, 432-449.

(2) Dias, P.; Vilanova, A.; Lopes, T.; Andrade, L.; Mendes, A. Extremely Stable Bare Hematite Photoanode for Solar Water Splitting. Nano Energy 2016, 23, 70-79.

(3) Kennedy, J. H.; Shinar, R.; Ziegler, J. P. $\alpha-\mathrm{Fe}_{2} \mathrm{O}_{3}$ Photoanodes Doped with Silicon. J. Electrochem. Soc. 1980, 127, 2307-2309.

(4) Launay, J. C.; Horowitz, G. Crystal Growth and Photoelectrochemical Study of Zr-Doped $\alpha-\mathrm{Fe}_{2} \mathrm{O}_{3}$ Single Crystal. J. Cryst. Growth 1982, 57, 118-124.

(5) Cherepy, N. J.; Liston, D. B.; Lovejoy, J. A.; Deng, H.; Zhang, J. Z. Ultrafast Studies of Photoexcited Electron Dynamics in $\gamma$ - and $\alpha-\mathrm{Fe}_{2} \mathrm{O}_{3}$ Semiconductor Nanoparticles. J. Phys. Chem. B 1998, 102, $770-776$.

(6) Kennedy, J. H.; Frese, K. W. Photooxidation of Water at $\alpha-\mathrm{Fe}_{2} \mathrm{O}_{3}$ Electrodes. J. Electrochem. Soc. 1978, 125, 709-714.

(7) Kennedy, J. H.; Anderman, M.; Shinar, R. Photoactivity of Polycrystalline $\alpha-\mathrm{Fe}_{2} \mathrm{O}_{3}$ Electrodes Doped with Group IVA Elements. J. Electrochem. Soc. 1981, 128, 2371-2373.

(8) Malviya, K. D.; Dotan, H.; Shlenkevich, D.; Tsyganok, A.; Mor, H.; Rothschild, A. Systematic Comparison of Different Dopants in Thin Film Hematite $\left(\alpha-\mathrm{Fe}_{2} \mathrm{O}_{3}\right)$ Photoanodes for Solar Water 
Splitting. J. Mater. Chem. A 2016, 4, 3091-3099.

(9) Green, M. A.; Emery, K.; Hishikawa, Y.; Warta, W.; Dunlop, E. D. Solar Cell Efficiency Tables (Version 47). Prog. Photovoltaics Res. Appl. 2016, 24, 3-11.

(10) Carlson, D. E.; Wronski, C. R. Amorphous Silicon Solar Cell. Appl. Phys. Lett. 1976, 28, 671.

(11) Würfel, U.; Würfel, U.; Cuevas, A.; Würfel, P. Charge Carrier Separation in Solar Cells. IEEE J. Photovoltaics 2015, 5, 461-469.

(12) Abdi, F. F.; Han, L.; Smets, A. H. M.; Zeman, M.; Dam, B.; van de Krol, R. Efficient Solar Water Splitting by Enhanced Charge Separation in a Bismuth Vanadate-Silicon Tandem Photoelectrode. Nat. Commun. 2013, 4, 2195.

(13) Li, J.; Meng, F.; Suri, S.; Ding, W.; Huang, F.; Wu, N.; Tilley, S.; Cornuz, M.; Silvula, K.; Grätzel, M.; et al. Photoelectrochemical Performance Enhanced by a Nickel Oxide-hematite P-n Junction Photoanode. Chem. Commun. 2012, 48, 8213-8215.

(14) Ahmed, M. G.; Kandiel, T. A.; Ahmed, A. Y.; Kretschmer, I.; Rashwan, F.; Bahnemann, D. Enhanced Photoelectrochemical Water Oxidation on Nanostructured Hematite Photoanodes via P$\mathrm{CaFe}_{2} \mathrm{O}_{4} / \mathrm{n}-\mathrm{Fe}_{2} \mathrm{O}_{3}$ Heterojunction Formation. J. Phys. Chem. C 2015, 119, 5864-5871.

(15) Lin, Y.; Xu, Y.; Mayer, M. T.; Simpson, Z. I.; McMahon, G.; Zhou, S.; Wang, D. Growth of P-Type Hematite by Atomic Layer Deposition and Its Utilization for Improved Solar Water Splitting. J. Am. Chem. Soc. 2012, 134, 5508-5511.

(16) Malviya, K. D.; Dotan, H.; Yoon, K. R.; Kim, I.-D.; Rothschild, A. Rigorous Substrate Cleaning Process for Reproducible Thin Film Hematite $\left(\alpha-\mathrm{Fe}_{2} \mathrm{O}_{3}\right)$ Photoanodes. J. Mater. Res. 2016, 31, 15651573.

(17) Engel, J.; Tuller, H. L. The Electrical Conductivity of Thin Film Donor Doped Hematite: From Insulator to Semiconductor by Defect Modulation. Phys. Chem. Chem. Phys. 2014, 16, 11374-11380.

(18) Kronik, L.; Shapira, Y. Surface Photovoltage Phenomena: Theory, Experiment, and Applications. Surf. Sci. Rep. 1999, 37, 1-206.

(19) Aphek, O. B.; Kronik, L.; Leibovitch, M.; Shapira, Y. Quantitative Assessment of the Photosaturation Technique. Surf. Sci. 1998, 409, 485-500.

(20) Saraf, S.; Rosenwaks, Y. Local Measurement of Semiconductor Band Bending and Surface Charge Using Kelvin Probe Force Microscopy; Surf.Sci. 2005, 574, 35-39.

(21) Krol, R.; Grätzel, M. Photoelectrochemical Hydrogen Production; Springer US, 2012.

(22) Rothschild, A.; Komem, Y. Numerical Computation of Chemisorption Isotherms for Device 
Modeling of Semiconductor Gas Sensors. Sensors Actuators B Chem. 2003, 93, 362-369.

(23) Grave, D. A.; Dotan, H.; Levy, Y.; Piekner, Y.; Scherrer, B.; Malviya, K. D.; Rothschild, A.; Sivula, K.; Formal, F. le; Grätzel, M.; et al. Heteroepitaxial Hematite Photoanodes as a Model System for Solar Water Splitting. J. Mater. Chem. A 2016, 4, 3052-3060.

(24) Zhao, B.; Kaspar, T. C.; Droubay, T. C.; McCloy, J.; Bowden, M. E.; Shutthanandan, V.; Heald, S. M.; Chambers, S. A. Electrical Transport Properties of Ti-Doped $\mathrm{Fe}_{2} \mathrm{O}_{3}$ (0001) Epitaxial Films. Phys. Rev. B 2011, 84, 245325.

(25) Turner, J. E.; Hendewerk, M.; Parmeter, J.; Neiman, D.; Somorjai, G. A. The Characterization of Doped Iron Oxide Electrodes for the Photodissociation of Water. J. Electrochem. Soc. 1984, 131, 1777-1783.

(26) Rettie, A. J. E.; Chemelewski, W. D.; Emin, D.; Mullins, C. B. Unravelling Small-Polaron Transport in Metal Oxide Photoelectrodes. J. Phys. Chem. Lett. 2016, 7, 471-479.

(27) Saraf, S.; Riess, I.; Rothschild, A. Parallel Band and Hopping Electron Transport in $\mathrm{SrTiO}_{3}$. Adv. Electron. Mater. 2016, 2, 1500368.

(28) Ma, G.; Suzuki, Y.; Singh, R. B.; Iwanaga, A.; Moriya, Y.; Minegishi, T.; Liu, J.; Hisatomi, T.; Nishiyama, H.; Katayama, M.; et al. Photoanodic and Photocathodic Behaviour of $\mathrm{La}_{5} \mathrm{Ti}_{2} \mathrm{CuS}_{5} \mathrm{O}_{7}$ Electrodes in the Water Splitting Reaction. Chem. Sci. 2015, 6, 4513-4518.

(29) Steier, L.; Luo, J.; Schreier, M.; Mayer, M. T.; Sajavaara, T.; Grätzel, M. Low-Temperature Atomic Layer Deposition of Crystalline and Photoactive Ultrathin Hematite Films for Solar Water Splitting. ACS Nano 2015, 9, 11775-11783.

(30) Dotan, H.; Sivula, K.; Grätzel, M.; Rothschild, A.; Warren, S. C.; Fujishima, A.; Honda, K.; Sanchez, C.; Sieber, K. D.; Somorjai, G. A.; et al. Probing the Photoelectrochemical Properties of Hematite $(\alpha-$ $\mathrm{Fe}_{2} \mathrm{O}_{3}$ ) Electrodes Using Hydrogen Peroxide as a Hole Scavenger. Energy Environ. Sci. 2011, 4 , 958-964.

(31) Glasscock, J. A.; Barnes, P. R. F.; Plumb, I. C.; Savvides, N. Enhancement of Photoelectrochemical Hydrogen Production from Hematite Thin Films by the Introduction of Ti and Si. J. Phys. Chem. C 2007, 111, 16477-16488.

(32) Bohn, C. D.; Agrawal, K. A.; Walter, E. C.; Vaudin, M. D.; Herzing, A. A.; Haney, P. M.; Talin, A. A.; Szalai, V. A. Effect of Tin Doping on $\alpha-\mathrm{Fe}_{2} \mathrm{O}_{3}$ Photoanodes for Water Splitting. J. Phys. Chem. C 2012, 116, 15290-15296. 